\title{
Manejo diagnóstico-terapéutico de las lesiones mamarias atípicas
}

\section{Diagnostic-therapeutic Management of Atypical Breast Lesions}

\author{
M. Delgado Márquez ${ }^{1}$ J. Rodríguez Arango ${ }^{2}$ \\ ${ }^{1}$ Servicio de Radiodiagnóstico, Hospital Universitario 12 de Octubre, \\ Madrid, España \\ 2 Servicio de Radiodiagnóstico, Hospital MD Anderson Cancer Center, \\ Madrid, España \\ Address for correspondence M. Delgado Márquez, MD, \\ Hospital Universitario 12 de Octubre, Madrid, España \\ (e-mail: manueldelgado80@hotmail.com). \\ Rev Argent Radiol 2018;82:114-123.
}

\section{Resumen \\ Palabras clave \\ - hiperplasia ductal atípica \\ - cicatriz radial \\ - neoplasia lobular \\ - tumor phyllodes \\ - linfoma mamario}
Abstract
Keywords
- atypical duct hiperplasia
- radial scar
- lobular neoplasia
- phyllodes tumor
- breast lymphoma

Las lesiones mamarias se dividen histológicamente en dos grandes grupos, malignas y benignas. Las lesiones malignas pueden ser de origen ductal o lobulillar, siendo el carcinoma ductal infiltrante la neoplasia invasiva más frecuente. Las lesiones benignas se clasifican en no proliferativas, proliferativas sin atipias y proliferativas con atipias. Dentro de los dos últimos grupos se encuentran entidades que conllevan un alto riesgo de desarrollar carcinoma de mama, como pueden ser la hiperplasia ductal atípica, la cicatriz radial o la neoplasia lobular. Revisamos en qué consisten dichas entidades y cuáles son sus características principales en imagen, fundamentalmente en mamografía y ecografía. Si tras realizar una biopsia se obtiene uno de esos diagnósticos histológicos, es importante analizar las características imagenológicas y el tipo de procedimiento realizado (número de cilindros obtenidos, calibre de aguja...), para realizar un adecuado manejo posterior. En algunos casos la actitud a seguir será la extirpación quirúrgica completa de la lesión, mientras que en otros se podrá realizar una extirpación percutánea (mediante biopsia con aguja de vacio), o incluso seguimiento estricto por imagen. Mediante diferentes casos mostraremos nuestra experiencia y analizaremos la literatura vigente para recordar esas entidades y llegar a un consenso adecuado sobre el manejo de las mismas.

Breast lesions are divided histologically into two large groups, malignant and benign. Malignant lesions may be of ductal or lobular origin, with infiltrating ductal carcinoma being the most frequent invasive neoplasm. Benign breast lesions are classified as proliferative, proliferative without atypia and proliferative with atypia. Within the last two classifications are entities that carry a high risk of developing breast carcinoma, such as atypical ductal hyperplasia, radial scar or lobular neoplasia. We review what these entities consist of and what are their fundamental characteristics in image, fundamentally in mammography and ultrasound. When we perform one of these histological diagnoses after a biopsy, it is important to analyze the radiological characteristics and the type of procedure performed (number of cylinders, needle received

October 16, 2017

accepted

February 25, 2018

published online

April 10, 2018
DOI https://doi.org/

$10.1055 / \mathrm{s}-0038-1641135$.

ISSN 1852-9992.
Copyright ( 2 2019, Sociedad Argentina de Radiología. Publicado por Thieme Revinter Publicações Ltda., Rio de Janeiro, Brazil. Todos los derechos reservados.

\section{License terms}

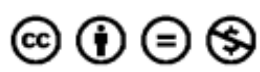


gauge ...) to perform an appropriate posterior management. In some cases the attitude to be followed will be the complete surgical removal of the lesion, while in others a percutaneous excision (through vacuum needle biopsy) or even strict image follow-up may be performed. Through different cases we will show our experience and analyze current literature to remember these entities and reach an adequate management consensus.

\section{Introducción}

Las lesiones mamarias benignas se clasifican en tres grupos según sus características histopatológicas. ${ }^{1-5}$

1. Lesiones no proliferativas. Esas entidades no se asocian con un mayor riesgo de carcinoma de mama. ${ }^{1}$ Dentro de ese grupo entrarían los quistes simples, las calcificaciones epiteliales, los galactoceles y lesiones de extirpe grasa como los lipomas y las necrosis grasas.

2. Lesiones proliferativas sin atipia. Esas entidades conllevan un leve aumento del riesgo de desarrollar carcinoma, aproximadamente de 1,5 a 2 veces con respecto a la población en general. ${ }^{2-5}$ Aquí encontraríamos a entidades como la hiperplasia ductal usual, el papiloma intraductal, la adenosis esclerosante y la cicatriz radial. Los fibroadenomas también entrarían en esa clasificación, ${ }^{6}$ pero sólo en el caso de que muestren una estructura histológica compleja, se asocien a enfermedad proliferativa adyacente o la paciente tenga una historia familiar de cáncer de mama.

3. Lesiones proliferativas con atipia. Se incluyen lesiones como la hiperplasia ductal atípica, la neoplasia lobular (que engloba hiperplasia lobulillar atípica y carcinoma lobulillar in situ) y la atipia epitelial plana. El riesgo de desarrollar carcinoma se multiplica de tres a cinco veces con respecto a la población general, pudiendo incluso indicar ese diagnóstico, la existencia de malignidad concomitante. ${ }^{7}$

4. Miscelánea. Dentro de las lesiones atípicas mamarias encontramos un cuarto grupo donde encontramos entidades como el linfoma mamario u otras lesiones benignas como el tumor de células granulares, afectaciones inflamatorias y tumores mesenquimales.

Hoy en día en un servicio de radiodiagnóstico es muy frecuente la realización de biopsias sobre lesiones mamarias visibles en mamografía, ecografía, o incluso resonancia magnética. En muchas ocasiones, las pruebas de imagen se realizan de manera rutinaria en pacientes asintomáticas (screening), mientras que otras son estudios motivados por alguna sintomatología como puede ser: molestia, palpación de nódulo, secreción por el pezón, etc.

Cuando realizamos una biopsia con aguja gruesa (BAG), ésas pueden ser de disparo automático o semiautomático, y con un calibre que va desde 11 a 16 Gauge, permitiendo diferentes tamaños de muestra. Se realizan varios pases a través de un único orificio en la piel de la paciente, obteniéndose una media de cuatro a cinco cilindros por biopsia.

Al realizar una biopsia con aguja de vacío (BAV), el sistema, además de cortar, realiza un aspirado de la lesión, obteniéndose cilindros de mayor tamaño con una muestra más representativa (de nueve a once Gauge). En general con una única entrada a través de la piel se obtienen varias muestras que se acumulan en una celdilla en la parte posterior de la aguja. La media de cilindros obtenidos es de ocho a doce, reservándose especialmente ese tipo de biopsia para el estudio de microcalcificaciones. Cuando al realizar una biopsia mamaria el resultado que obtenemos es el de una lesión atípica, es muy importante valorar qué tipo de sistema de biopsia hemos empleado, el número de cilindros obtenidos y el tipo de lesión sobre el que hemos realizado el estudio (nódulo, calcificaciones, distorsión, etc.). También debemos asegurar que se ha efectuado un adecuado procesamiento de la muestra en el servicio de anatomía patológica.

Dado que la aguja únicamente extrae cierta cantidad de tejido y se puede subestimar el hallazgo radiológico, cuando obtenemos uno de esos resultados, es importante realizar una correlación entre la imagen radiológica y el resultado histológico para decidir la actitud a seguir, preferiblemente en un Comité Multidisciplinar donde la decisión sea consensuada.

En el presente trabajo vamos a revisar algunas de las lesiones atípicas más frecuentes mediante ejemplos, así como el manejo más adecuado de las mismas según una revisión de la literatura.

\section{Lesiones Papilares}

Las lesiones papilares de la mama (-Fig. 1) son proliferaciones arborescentes del epitelio ductal, con un pedículo fibrovascular. Se desarrollan en la luz adheridas a las paredes en cualquier parte del sistema ductal, desde el pezón hasta la unidad ducto-lobulillar más periférica. Representan menos de un 3\% de las lesiones mamarias sólidas y pueden ser solitarias o múltiples. Según su clasificación histológica se dividen en benignas, atípicas y malignas; incluyendo dentro de esa última, al carcinoma intraductal y al carcinoma papilar invasor.

Según diferentes estudios, aproximadamente un $15 \%$ de las lesiones que han sido diagnosticadas mediante biopsia con aguja gruesa (BAG) con resultado de papilomas benignos, pasan a ser atípicos o malignos tras la extirpación quirúrgica 


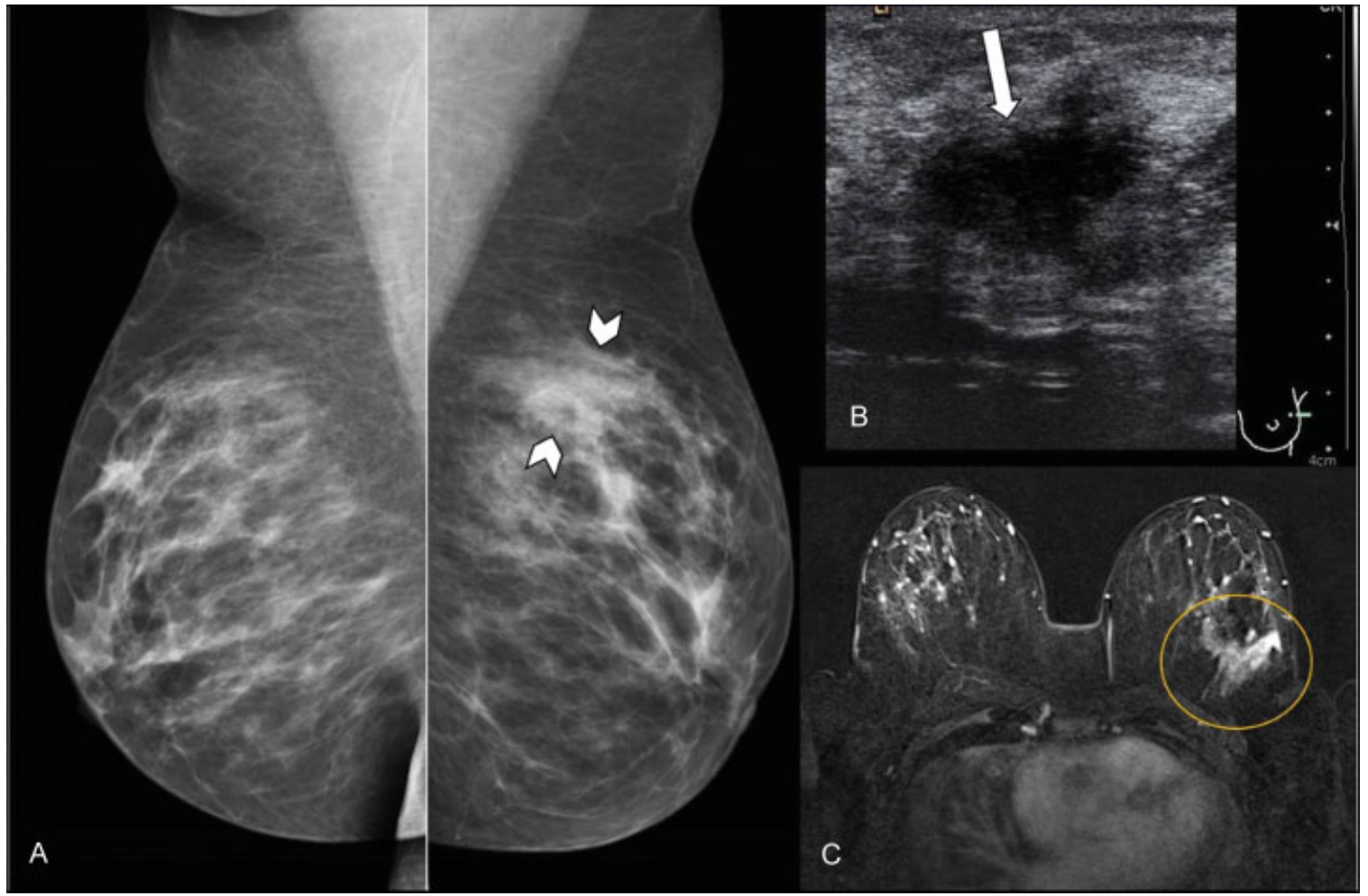

Fig. 1 Papilomatosis florida. Mujer de 52 años, que acudió a consulta por induración en mama izquierda. (A) Mamografía digital. Asimetría focal con mayor densidad en cuadrante superior externo (CSE) de mama izquierda (puntas de flecha). (B) Ecografía. Nódulo hipoecoico de morfología irregular (flecha). (C) Resonancia magnética (RM) con contraste intravenoso. Realce de contraste en CSE de mama izquierda, no masa, de distribución regional (círculo). El resultado histológico tras biopsia con aguja gruesa (BAG) fue de papilomas múltiples. Se realizó una extirpación quirúrgica, con resultado final de papilomatosis florida con hiperplasia ductal atípica.

y posterior estudio anatomopatológico, siendo ese riesgo mayor si la paciente presenta una papilomatosis múltiple. ${ }^{8}$

Por tanto, en dicha situación, el manejo adecuado es la resección quirúrgica de la lesión completa, aunque cada vez existen más estudios que se inclinan por realizar una BAV sobre la lesión previamente biopsiada, con fin extirpativo. Cuando el resultado de la BAG es de papiloma atípico o maligno, la actitud a seguir es la de extirpación quirúrgica de la lesión mediante cirugía. ${ }^{8-12}$

\section{Cicatriz Radial}

También recibe el nombre de lesión esclerosante compleja, si es mayor de $10 \mathrm{~mm}$. Es una entidad de etiología desconocida, más frecuente en mujeres de los cuarenta a los sesenta años. Suele ser un hallazgo incidental, no palpable, descubierto en una revisión rutinaria, en la mayoría de ocasiones al realizar una mamografía de screening.

Tanto radiológica como histológicamente es una lesión espiculada (-Fig. 2). Consta de un tejido fibroelástico central, mientras que las espículas contienen estructuras ductales "atrapadas," normalmente con hiperplasia y/o metaplasia apocrina. Puede asociarse a atipia o a carcinoma. En el análisis histológico de la lesión completa, aproximadamente un 10\% muestran carcinoma asociado, permaneciendo todavía en duda si es una lesión premaligna. ${ }^{13}$
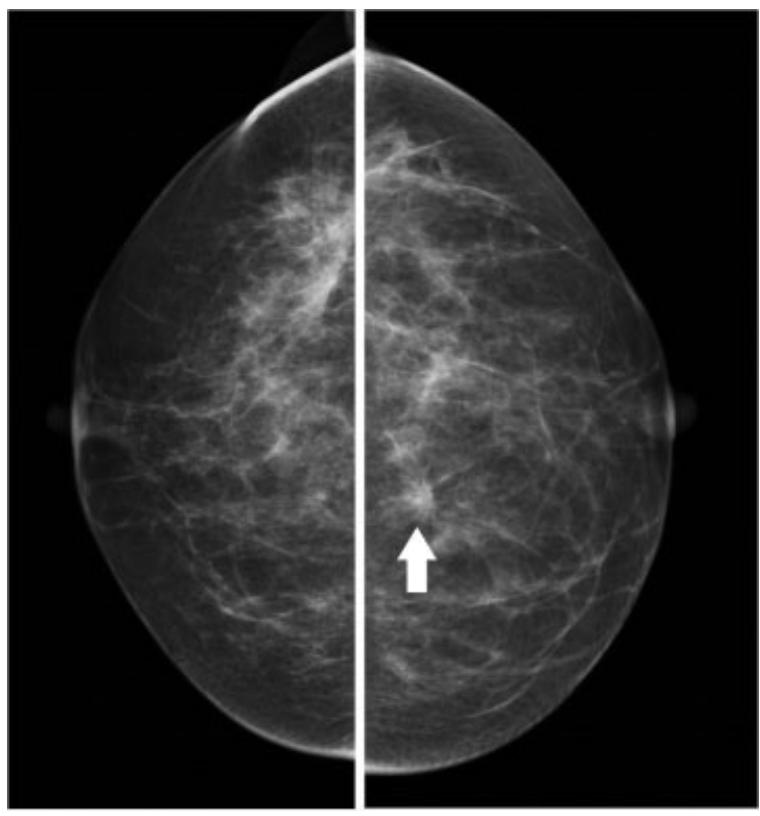

Fig. 2 Cicatriz radial. Mujer de 48 años, asintomática. Mamografía digital. En cuadrantes internos de mama izquierda se identificó una lesión espiculada junto con distorsión parenquimatosa (flecha), criterios sospechosos de malignidad. Se realizó BAG y posteriormente extirpación quirúrgica, con resultado de cicatriz radial. 
El riesgo de desarrollar un carcinoma mamario es aproximadamente el doble que en la población general (en cualquiera de las dos mamas). Por tanto, si al realizar una biopsia el resultado anatomopatológico es de cicatriz radial, el manejo adecuado es su extirpación quirúrgica completa.

\section{Atipia Epitelial Plana}

Esa entidad se encuentra dentro del espectro de lesiones columnares, que son alteraciones intraductales con proliferación celular. Cuando el informe histológico es de hiperplasia de células columnares (-Fig. $\mathbf{3}$ ), no es necesario realizar un tratamiento adicional. Cuando obtenemos un resultado de atipia epitelial plana (-Fig. 4), se está refiriendo a una hiperplasia columnar con células con atipia leve. Se clasificaría dentro de las lesiones DIN 1 A (neoplasia intraepitelial ductal).

El hallazgo radiológico más frecuente es el de microcalcificaciones, fundamentalmente amorfas. Puede coexistir o ser precursor de carcinoma intraductal. En los estudios realizados, el porcentaje de infraestimación es de aproximadamente un $20 \%$, es decir, en una de cada cinco pacientes en las cuales se extirpa quirúrgicamente la lesión por completo, encontraremos carcinoma mamario. ${ }^{14}$

El consenso a seguir debería ser de extirpación quirúrgica completa de la lesión, sobre todo si existe representación ecográfica. Si no quedan microcalcificaciones residuales tras

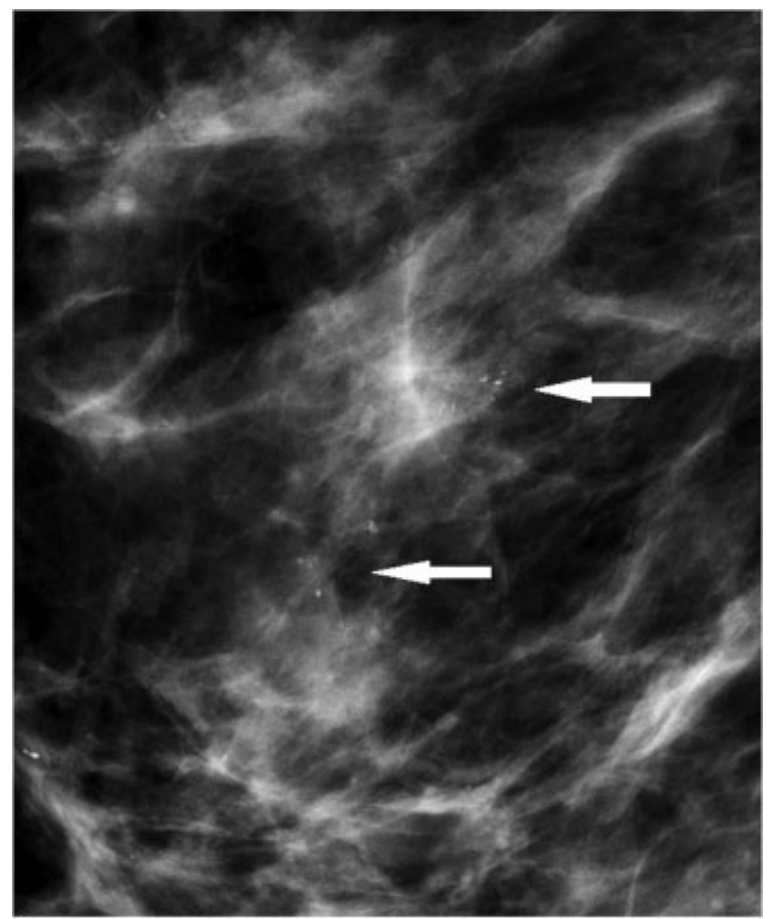

Fig. 3 Hiperplasia columnar. Mujer de 62 años, asintomática. Mamografía digital magnificada. Cúmulo de microcalcificaciones heterogéneas groseras (flechas), de nueva aparición comparando con estudios previos. Se realizó biopsia con aguja de vacío (BAV) con guía esterotáxica, con resultado de microcalcificacones asociadas a tejido mamario benigno con cambio columnar e hiperplasia columnar.

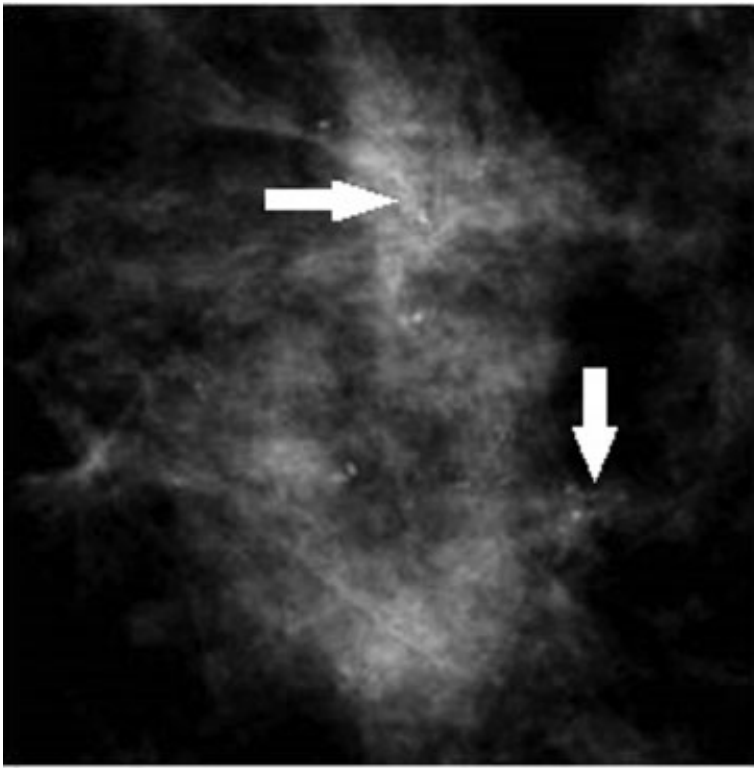

Fig. 4 Atipia epitelial plana. Mujer de 51 años, asintomática. Mamografía digital magnificada. Cúmulo de microcalcificaciones amorfas (flechas), que aumentaron en número comparando con estudios previos. Se realizó BAV con guía esterotáxica, con resultado de atipia epitelial plana. Posteriormente se realizó biopsia quirúrgica tras colocación de arpón con resultado de atipia epitelial plana y papilomatosis múltiple intraductal.

la realización de BAV, se podría optar por seguimiento estrecho mediante imagen.

\section{Hiperplasia Ductal Atípica}

Es la lesión benigna de alto riesgo más común. Consiste en la proliferación de células atípicas que ocupan parte del ducto. Histológicamente es similar al carcinoma intraductal de bajo grado, dependiendo si se trata de una entidad $u$ otra en función del número de ductos comprometidos y de la extensión del área afectada. ${ }^{15-18}$ Multiplica entre cuatro a cinco veces el riesgo de desarrollar carcinoma invasor para ambas mamas, siendo el riesgo por seis si la paciente es premenopaúsica. ${ }^{15}$ Incluso está en duda si en esas pacientes, dado su riesgo, estaría indicado el realizar seguimiento con resonancia magnética ( $R M)$, aunque no existen en la actualidad datos suficientes para recomendarlo. También se ha planteado el tratamiento farmacológico para reducir el riesgo mediante moduladores de los receptores de estrógenos (tamoxifeno / raloxifeno) y/o los inhibidores de la aromatasa. ${ }^{18}$

Radiológicamente la presentación más común es la de un cúmulo de microcalcificaciones heterogéneas (-Fig. 5), aunque también puede presentarse como un nódulo. La probabilidad de que resulte una lesión histológica de mayor grado en la cirugía es de aproximadamente un $87 \%$ si se ha realizado una BAG con calibre $14 \mathrm{G}$ y de un $15-30 \%$ si se ha realizado una BAV con calibre $9 \mathrm{G}$, aún cuando se ha realizado extirpación completa de la lesión visible en imagen (mamografía o ecografía). ${ }^{16,17}$ 


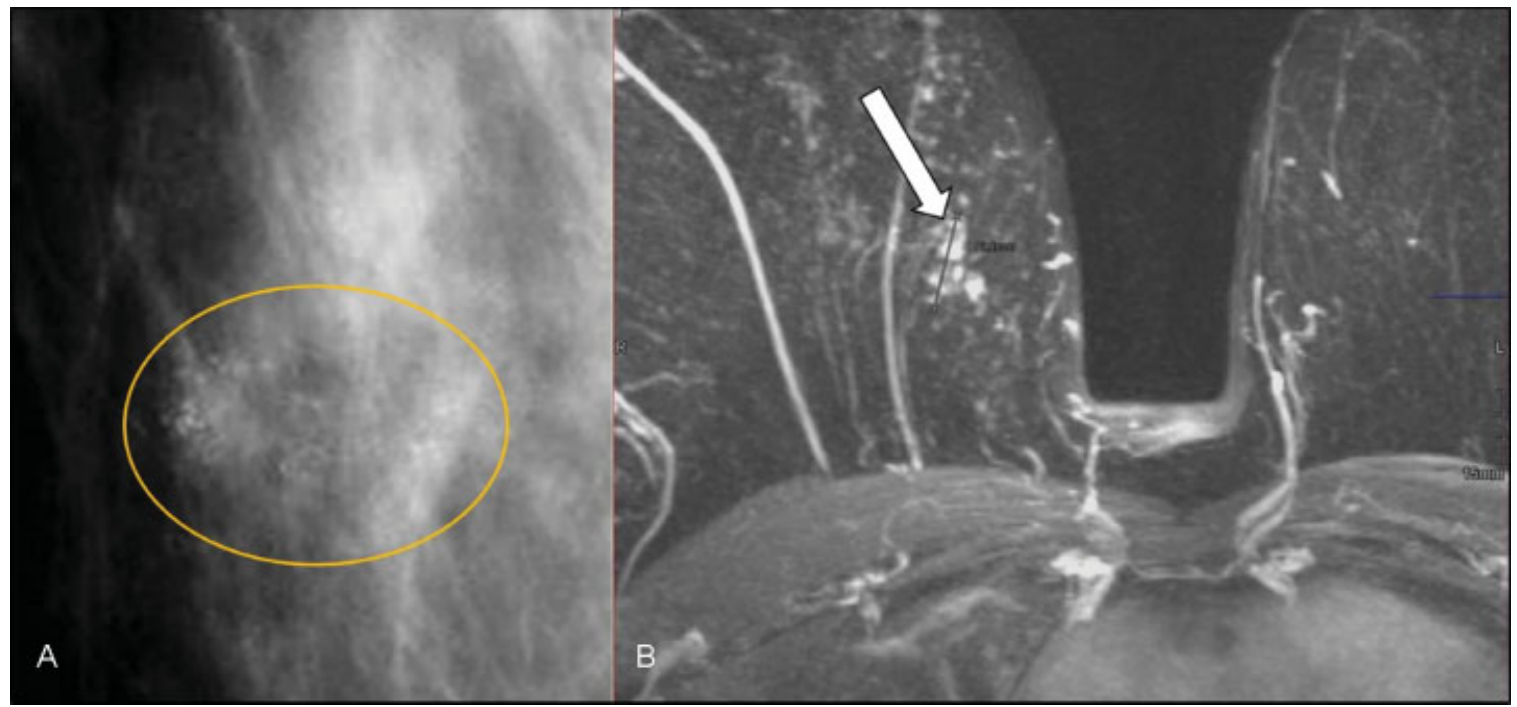

Fig. 5 Hiperplasia ductal atípica y carcinoma intraductal. Mujer de 72 años, asintomática. Mamografía digital. (A) Cúmulo de microcalcificaciones amorfas y lineales finas (delimitadas por círculo amarillo). (B) RM tras introducir contraste intravenoso. Realce con distribución focal, en empedrado que coincidió con las microcalcificaciones descritas, criterios sospechosos de malignidad (flecha). Se realizó BAV con guía esterotáxica, con resultado de hiperplasia ductal atípica. Al realizar extirpación completa de las microcalcificaciones el resultado definitivo fue de carcinoma intraductal de alto grado.

Por todo lo anterior, el manejo adecuado de ese tipo de lesiones es la extirpación quirúrgica.

\section{Neoplasia Lobular}

Concepto que engloba a la hiperplasia lobulillar atípica (HLA) y al carcinoma lobulillar in situ (CLIS), siendo la diferencia fundamental la presencia o no de distensión de los acinos, hallazgo existente en el CLIS. Suelen ser entidades con distribución multifocal y bilateral.

No son precursores obligatoriamente de carcinoma de mama, pero sí son marcadores de riesgo para carcinoma invasor en ambas mamas (algo más en la mama ipsilateral). La hiperplasia lobulillar atípica multiplica por cuatro o cinco el riesgo de desarrollar carcinoma infiltrante, mientras que el carcinoma lobulillar in situ (- Fig. 6) lo hace por 8-10. ${ }^{19}$

Los hallazgos en imagen de esas entidades pueden ser calcificaciones o nódulos. No es infrecuente su asociación a otra entidad diferente y que se trate de un hallazgo incidental. Debido a su elevado riesgo de desarrollar un carcinoma infiltrante, al igual que con la hiperplasia ductal atípica, también se ha planteado el seguimiento con RM y el tratamiento médico con fármacos moduladores de los receptores de estrógenos $\mathrm{y} / \mathrm{o}$ con inhibidores de la aromatasa para intentar disminuir dicho riesgo.

El manejo adecuado de esa entidad es la extirpación quirúrgica de la lesión si hay congruencia radiopatológica y en especial ante un carcinoma lobulillar in situ cuya traducción en imagen es un nódulo. Tras la cirugía, el porcentaje de malignidad es de un $20 \%$. Existe una variante del CLIS, el tipo pleomórfico, que tiene un curso biológico más agresivo y se considera precursor de carcinoma intraductal. En ese caso, está indicada siempre la extirpación quirúrgica completa de la lesión.

\section{Tumor Phyllodes}

Neoplasia de origen fibroepitelial. Su origen está fuera de los ductos y lóbulos, en el tejido conectivo. Representa menos del $0,5 \%$ de los tumores de la mama. Se clasifican en benignos (58\%), potencialmente malignos o borderline (12\%) y malignos (30\%). Su presentación más habitual es como un nódulo sólido, palpable, de crecimiento rápido (-Fig. 7). Ecográficamente muestran una morfología redondeada o lobulada. Suelen tener una ecoestructura heterogénea aunque son fundamentalmente hipoecoicos. Presentan bordes bien definidos y buena transmisión del sonido.

En un 20\% de las ocasiones, esas pacientes presentan un fibroadenoma concomitante, y en un $12,5 \%$ existe una historia personal de fibroadenomas. ${ }^{20}$ El tratamiento adecuado es la extirpación quirúrgica, local, con un margen de 1-2 cm dado que son propensos a la recurrencia local. Si son de gran tamaño, habría que optar por mastectomía. No es necesario realizar linfadenectomía axilar, ya que también es rara la diseminación axilar.

\section{Fascitis Nodular}

Se trata de una lesión proliferativa benigna de fibroblastos y miofibroblastos, cuya localización más frecuente es en partes blandas del brazo, cabeza y cuello. Si se localiza en la mama, lo cual es muy infrecuente, su presentación es similar a la de un carcinoma ( - Fig. 8). Suele presentarse como un nódulo único de crecimiento rápido, duro y de morfología irregular. ${ }^{21}$

El manejo adecuado es la extirpación completa de la lesión sin tratamiento adicional, siendo baja la tasa de recidiva, habiéndose descrito en ocasiones incluso la desaparición espontánea de la lesión. 


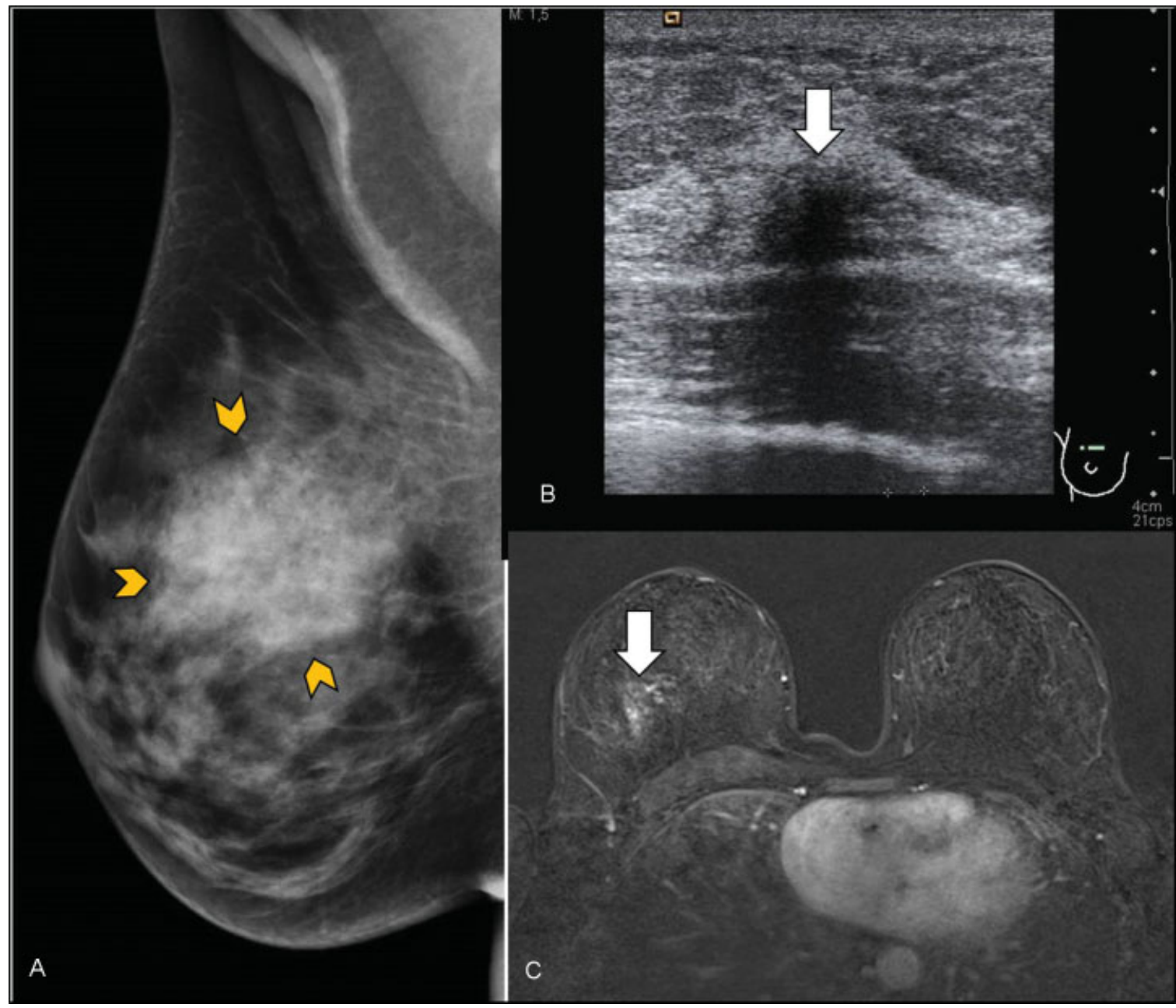

Fig. 6 Neoplasia lobulillar in situ. Mujer de 48 años que acudió por masa palpable en mama derecha. (A) Mamografía digital mama derecha. Aumento de densidad de morfología nodular en cuadrantes superiores (delimitado por puntas de flechas amarillas). (B) Ecografía. Nódulo hipoecoico de morfología irregular y bordes mal delimitados (flecha). (C) RM con contraste intravenoso. Se apreció un realce asimétrico, no masa, con distribución focal (flecha). Se realizó BAG con control ecográfico con resultado de neoplasia lobulillar in situ. Al realizar cirugía de la lesión completa, se obtuvo el mismo resultado.

\section{Tumor de Células Granulares}

Lesión infrecuente, con histogénesis incierta, cuyo origen parece encontrarse en las células de Schwann. Puede presentarse en cualquier lugar del cuerpo y a cualquier edad, siendo más frecuente en región de cabeza-cuello, especialmente en la lengua. ${ }^{22}$ Cuando se localiza en la mama, suele asentar en el cuadrante superior interno (CSI), dada su relación con el trayecto del nervio supraclavicular, que inerva la piel mamaria. Ese sería uno de sus criterios diferenciales respecto al carcinoma, cuya localización más frecuente es en el cuadrante superior externo (CSE).

Suele presentarse como una masa firme, indolora, que puede causar retracción cutánea e inversión del pezón, siendo imposible establecer el diagnóstico definitivo sin biopsia (—Fig. 9). La mayoría son benignos, siendo malignos aproximadamente el $2 \%$.

El tratamiento adecuado es su excisión local, no siendo rara la recidiva.

\section{Linfoma Mamario}

Representa el 0,5\% de todos los cánceres de mama, siendo el subtipo más frecuente el no Hodgkin, de células B. Existen dos entidades diferentes, indistinguibles en imagen y en estudio histológico: uno de ellos es el linfoma primario de la mama y otro una afectación linfomatosa secundaria, que es más frecuente.

Aproximadamente entre uno y dos por ciento de todos los linfomas aparecen en la mama, siendo más frecuentes en mujeres post menopáusicas. La afectación puede ser unilateral o bilateral. Puede presentarse como: a) una masa solitaria; b) múltiples masas; ${ }^{23,24}$ c) patrón infiltrativo (-Fig. 10), menos frecuente, que consta de un aumento de densidad generalizado del parénquima mamario y de la grasa en mamografía. Si nos encontramos con un patrón infiltrativo difuso, se debe hacer el diagnóstico diferencial entre varias entidades: linfoma mamario, carcinoma inflamatorio, mastitis o congestión venosa (por fallo cardiaco o compromiso del retorno venoso). 


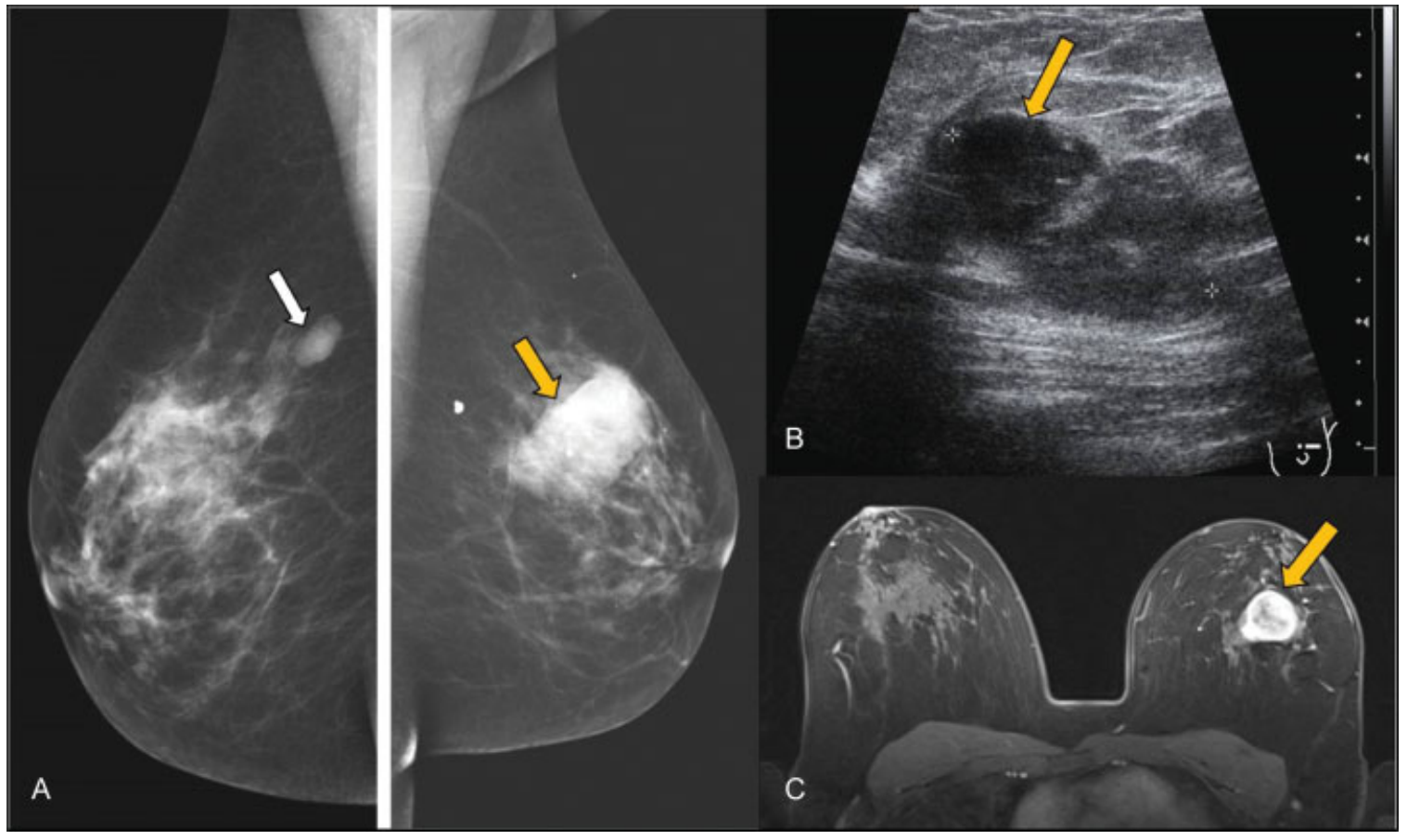

Fig. 7 Tumor Phyllodes. Mujer de 45 años con antecedente de fibroadenoma en mama derecha que acudió por nódulo palpable de nueva aparición en mama izquierda. (A) Mamografía digital. Nódulos bilaterales, el de mayor tamaño en cuadrante superior externo (CSE) de mama izquierda (flecha amarilla). (B) Ecográficamente es un nódulo sólido, de bordes lobulados y ecogenicidad heterogénea (flecha), con zonas de buena transmisión del sonido. (c) RM con contraste iv. Nódulo hipervascular de bordes bien definidos (flecha). Tanto en la BAG como en la extirpación completa, el resultado histológico es de Tumor Phyllodes.
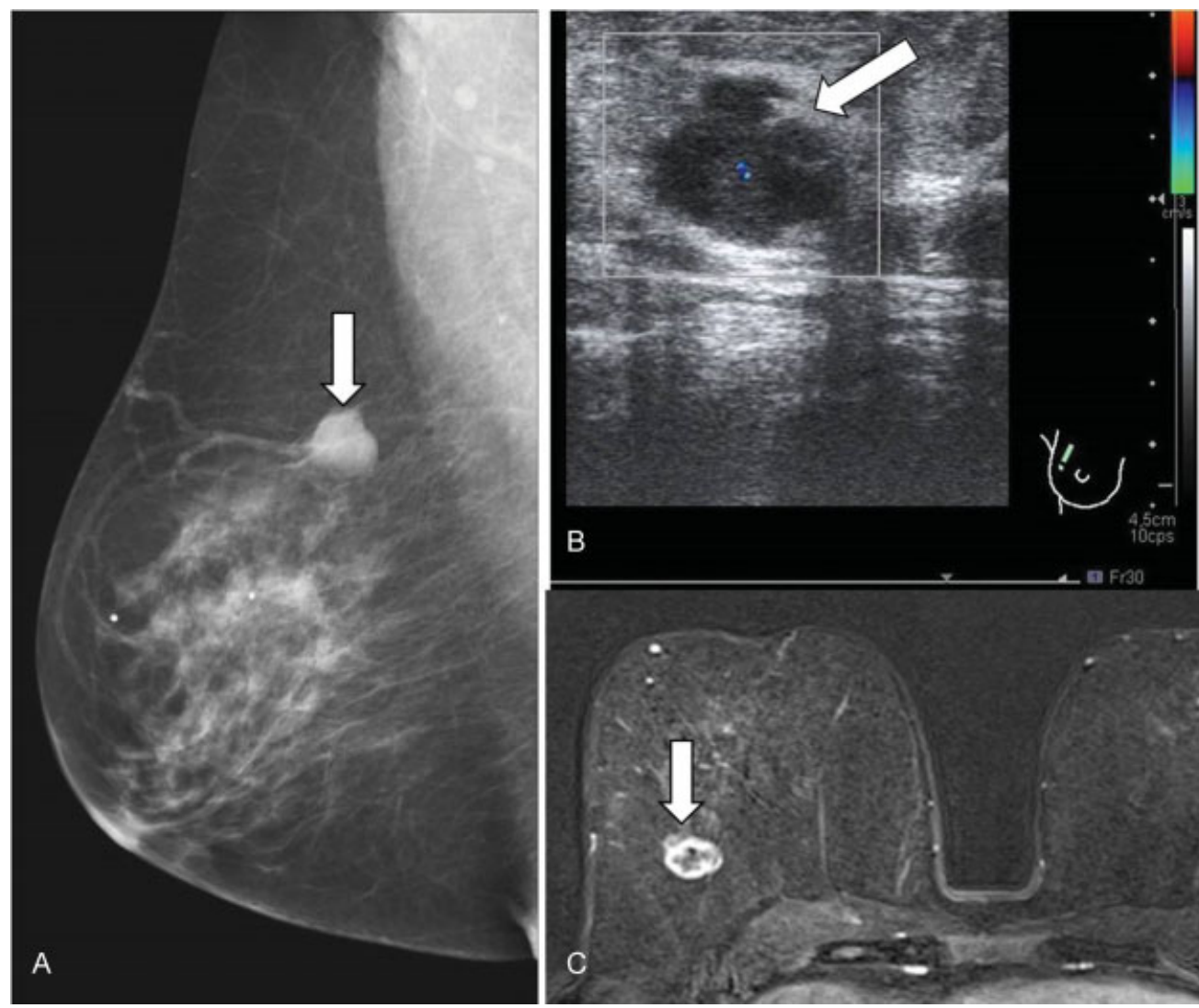

Fig. 8 Fascitis nodular. Paciente mujer de 52 años que refirió nódulo palpable de nueva aparición. (A) Mamografía digital. Nódulo en CSE de mama derecha (flecha). (B) Ecográficamente es un nódulo hipoecoico, de morfología redondeada aunque bordes mal definidos. (C) RM con contraste intravenoso. Nódulo sólido con captación en anillo con un centro hipointenso (flecha). Se realizó BAG con control ecográfico con resultado de tumoración fusocelular con atipia leve. Al extirpar el nódulo quirúrgicamente en su totalidad se obtuvo el diagnóstico final de fascitis nodular. 


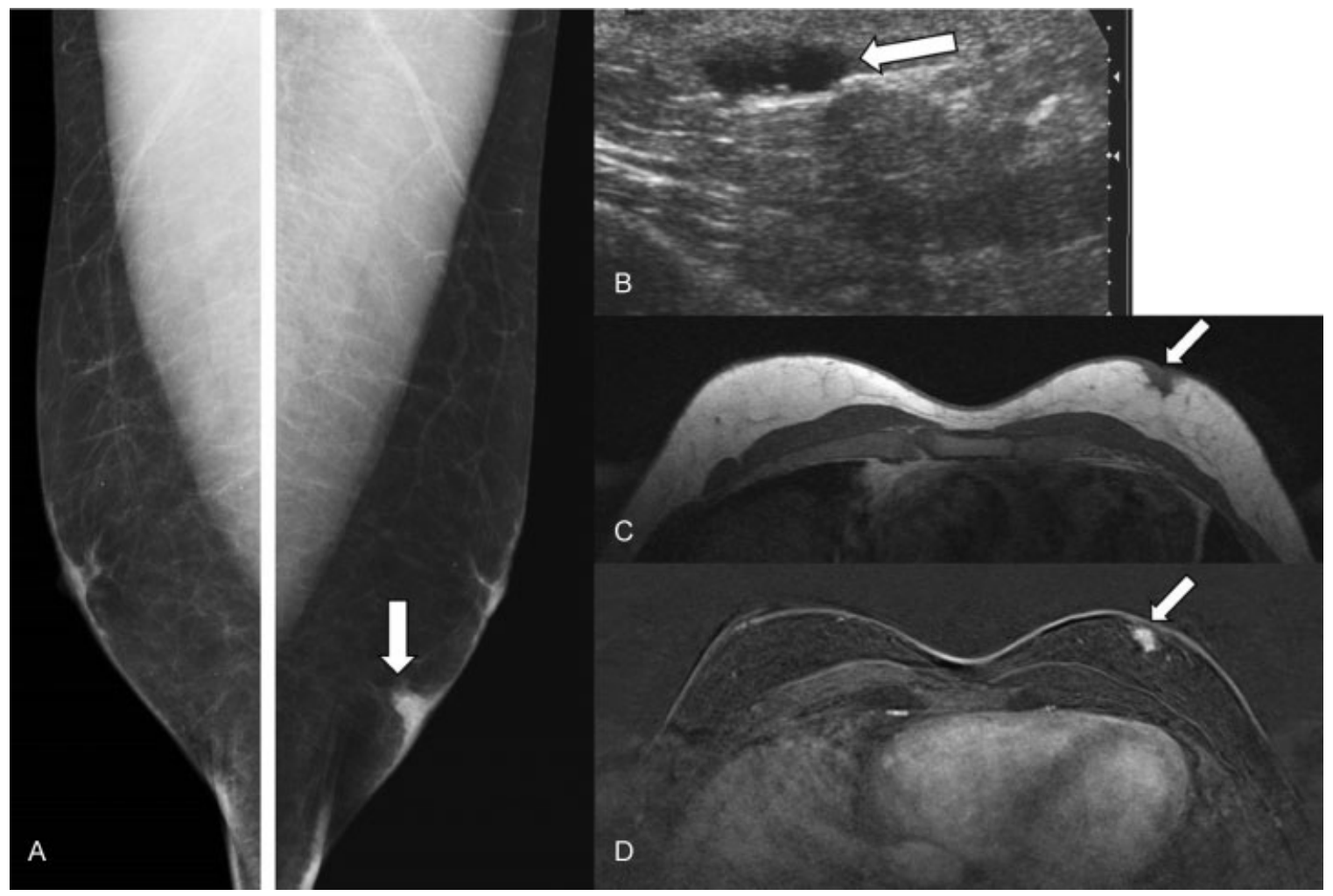

Fig. 9 Tumor de células granulares. Varón de 30 años que refirió un bulto de nueva aparición en cuadrantes inferiores de mama izquierda. (A) Mamografía digital. Se observó un nódulo superficial, con engrosamiento de la piel en cuadrantes inferiores de mama izquierda (flecha). (B) Ecografía. Nódulo hipoecoico, ovalado (flecha). (C-D) RM (secuencia ponderada en T1 sin administrar contraste intravenoso y postcontraste) donde se observó que dicho nódulo presenta bordes mal definidos y una captación precoz de contraste (flechas). El resultado histológico tras la biopsia y la extirpación quirúrgica fue de tumor de células granulares.
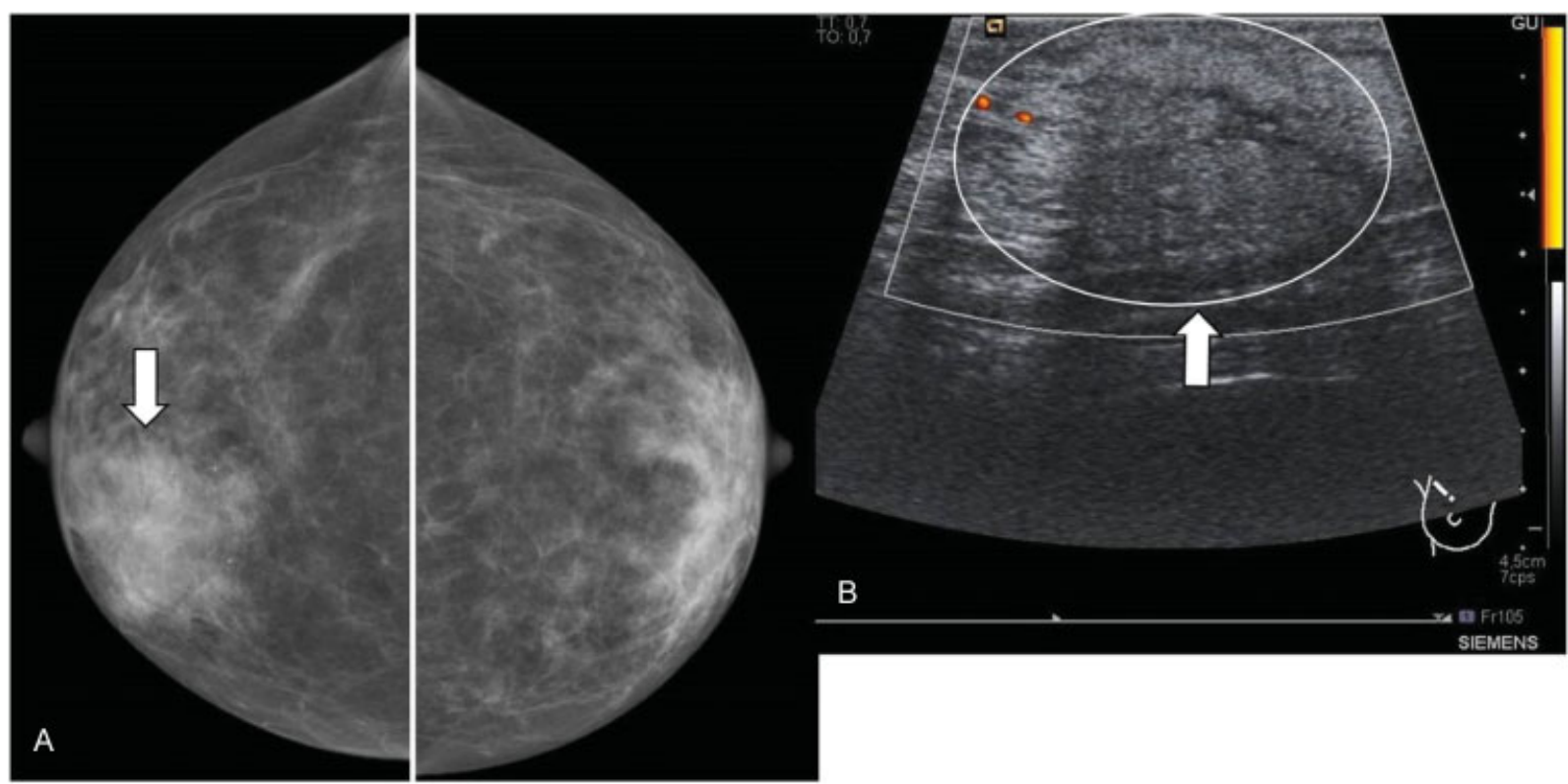

Fig. 10 Linfoma mamario. Paciente de 70 años que consultó por induración en región periareolar de mama derecha. (A) Mamografía digital. Se observó un aumento de densidad discretamente asimétrico, de morfología nodular, en región retroareolar de mama derecha. (B) Ecografía. Se identificó engrosamiento de la piel con hiperecogenicidad de la grasa subcutánea y pequeñas láminas de líquido (flecha y círculo). El resultado de la biopsia fue de infiltración por proceso linfoproliferativo B de bajo grado. 


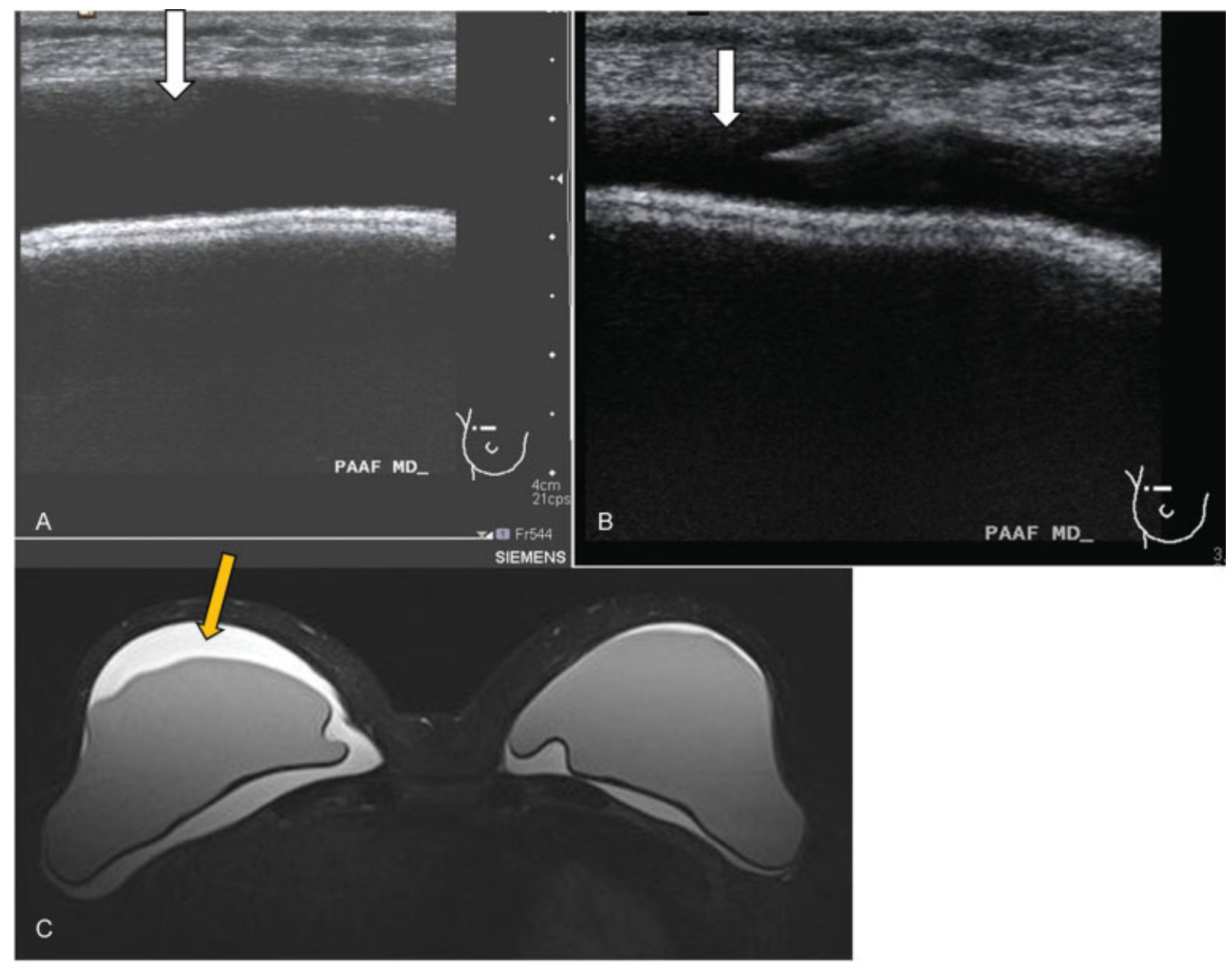

Fig. 11 Linfoma anaplásico asociado a prótesis. Paciente mujer de 55 años con antecedente de mastectomía bilateral por carcinoma lobulillar in situ. Refirió molestias en ambas mamas, fundamentalmente en el lado derecho. (A) Ecografía. Líquido anecoico periprotésico de nueva aparición, de predominio en el lado derecho (flecha). (B) Se realizó punción aspiración con aguja fina (PAAF) con control ecográfico del líquido (flecha). (C) RM secuencia potenciada en T2 donde se identificó líquido periprotésico bilateral, de predominio en el lado derecho (flecha amarilla) e integridad de las prótesis en secuencias específicas (no incluidas). El resultado citológico de la punción fue de linfoma anaplásico de célula grande asociado a implante mamario.

\section{Linfoma Anaplásico de Células Grandes}

Es una entidad muy rara, existiendo aproximadamente 200 casos publicados, 6 de ellos bilaterales. Se trata de un linfoma de células T asociado a prótesis (CD30-, ALK-). La forma más frecuente de presentación es la de un seroma periprótesis tardío ( + de 1 año), sin otra causa que lo justifique como podría ser traumatismo o infección (-Fig. 11).

Su causa es desconocida, aparentemente por una reacción inmunológica exagerada. Para diagnosticarlo es necesario realizar una punción aspiración con aguja fina (PAAF), con control ecográfico $\mathrm{y}$ análisis citológico del líquido periprótesis. $^{25}$ Una vez obtenido ese diagnóstico, el tratamiento adecuado es retirar la prótesis y capsulectomía completa. En la mayoría de casos no es necesario quimioterapia ni radioterapia.

Por tanto, como recomendación, se debe realizar PAAF ante el hallazgo de un seroma periprótesis de inicio brusco sin otra causa que lo justifique, para descartar esa entidad.

\section{Conclusiones}

Con la implantación de los programas de screening mamario y el amplio número de biopsias que se realizan hoy en día en los servicios de radiología, cada vez es más frecuente obtener un diagnóstico histológico de una lesión proliferativa, bien sea con o sin atipias.

El manejo de las lesiones mamarias atípicas es un tema controvertido dado que muchas de ellas se asocian a malignidad en un porcentaje elevado mientras que otras son un marcador de riesgo para desarrollar carcinoma. Por ello es importante analizar las características radiológicas y el tipo de procedimiento realizado (número de cilindros obtenidos, calibre de aguja...) para realizar un adecuado manejo posterior.

En algunos casos la actitud a seguir será la extirpación quirúrgica completa de la lesión, mientras que en otros se podrá realizar una extirpación percutánea (mediante biopsia con aguja de vacio) o incluso seguimiento estricto por imagen. 
Conflicto de Intereses

Los autores declaran no tener ningún conflicto de interés.

\section{Responsabilidades Éticas}

Protección de personas y animales. Los autores declaran que para esta investigación no se han realizado experimentos en seres humanos ni en animales.

Confidencialidad de los datos. Los autores declaran que han seguido los protocolos de su centro de trabajo sobre la publicación de datos de pacientes.

Derecho a la privacidad y consentimiento informado. Los autores declaran que en este artículo no aparecen datos de pacientes.

\section{Bibliografía}

1 Schnitt SJ. Benign breast disease and breast cancer risk: morphology and beyond. Am J Surg Pathol 2003;27(06):836-841

2 London SJ, Connolly JL, Schnitt SJ, Colditz GA. A prospective study of benign breast disease and the risk of breast cancer. JAMA 1992; 267(07):941-944

3 Dupont WD, Page DL. Risk factors for breast cancer in women with proliferative breast disease. N Engl J Med 1985;312(03):146-151

4 Hartmann LC, Sellers TA, Frost MH, et al. Benign breast disease and the risk of breast cancer. N Engl J Med 2005;353(03):229-237

5 Schnitt SJ, Collins LC. Pathology of benign breast disorders. In: Breast diseases, Harris JR, et al (Eds), Lippincott2010. p.69

6 Guray M, Sahin AA. Benign breast diseases: classification, diagnosis, and management. Oncologist 2006;11(05):435-449

7 Degnim AC, Visscher DW, Berman HK, et al. Stratification of breast cancer risk in women with atypia: a Mayo cohort study. J Clin Oncol 2007;25(19):2671-2677

8 Wen X, Cheng W. Nonmalignant breast papillary lesions at coreneedle biopsy: a meta-analysis of underestimation and influencing factors. Ann Surg Oncol 2013;20(01):94-101

9 Lewis JT, Hartmann LC, Vierkant RA, et al. An analysis of breast cancer risk in women with single, multiple, and atypical papilloma. Am J Surg Pathol 2006;30(06):665-672

10 Valdes EK, Feldman SM, Boolbol SK. Papillary lesions: a review of the literature. Ann Surg Oncol 2007;14(03):1009-1013

11 Mercado CL, Hamele-Bena D, Oken SM, Singer CI, Cangiarella J. Papillary lesions of the breast at percutaneous core-needle biopsy. Radiology 2006;238(03):801-808
12 Sydnor MK, Wilson JD, Hijaz TA, Massey HD, Shaw de Paredes ES. Underestimation of the presence of breast carcinoma in papillary lesions initially diagnosed at core-needle biopsy. Radiology 2007; 242(01):58-62

13 Manfrin E, Remo A, Falsirollo F, Reghellin D, Bonetti F. Risk of neoplastic transformation in asymptomatic radial scar. Analysis of 117 cases. Breast Cancer Res Treat 2008;107(03):371-377

14 Schnitt SJ. The diagnosis and management of pre-invasive breast disease: flat epithelial atypia-classification, pathologic features and clinical significance. Breast Cancer Res 2003;5(05):263-268

15 Wagoner MJ, Laronga C, Acs G. Extent and histologic pattern of atypical ductal hyperplasia present on core needle biopsy specimens of the breast can predict ductal carcinoma in situ in subsequent excision. Am J Clin Pathol 2009;131(01):112-121

16 Ko E, Han W, Lee JW, et al. Scoring system for predicting malignancy in patients diagnosed with atypical ductal hyperplasia at ultrasound-guided core needle biopsy. Breast Cancer Res Treat 2008;112(01):189-195

17 Menes TS, Rosenberg R, Balch S, Jaffer S, Kerlikowske K, Miglioretti DL. Upgrade of high-risk breast lesions detected on mammography in the Breast Cancer Surveillance Consortium. Am J Surg 2014;207(01):24-31

18 Visvanathan K, Chlebowski RT, Hurley P, et al; American Society of Clinical Oncology. American society of clinical oncology clinical practice guideline update on the use of pharmacologic interventions including tamoxifen, raloxifene, and aromatase inhibition for breast cancer risk reduction. J Clin Oncol 2009;27 (19):3235-3258

19 Morrow M, Schnitt SJ. Lobular carcinoma in situ. In: Diseases of the Breast, Harris JR, Lippman ME, Morrow M, Hellman S (Eds), Philadelphia: Lippincott-Raven; 1995. p.369

20 Wurdinger $S$, Herzog $A B$, Fischer DR, et al. Differentiation of phyllodes breast tumors from fibroadenomas on MRI. AJR Am J Roentgenol 2005;185(05):1317-1321

21 A Thomas Stavros. Ecografía de mama. Madrid: Marban; 2007

22 Brown AC, Audisio RA, Regitnig P. Granular cell tumour of the breast. Surg Oncol 2011;20(02):97-105

23 Meyer JE, Kopans DB, Long JC. Mammographic appearance of malignant lymphoma of the breast. Radiology 1980;135(03): 623-626

24 Lyons JA, Myles J, Pohlman B, Macklis RM, Crowe J, Crownover RL. Treatment of prognosis of primary breast lymphoma: a review of 13 cases. Am J Clin Oncol 2000;23(04):334-336

25 van der Veldt AA, Kleijn SA, Nanayakkara PW. Silicone breast implants and anaplastic large T-cell lymphoma. JAMA 2009;301 (12):1227, author reply 1227 